\title{
Intrauterine Subclinical Inflammation Sensitizes Hypoxic Ischemia- Induced Injury in the Immature Rat Brain and the Mechanisms
}

Xu Fa-lin',2*, Zhang Yan-hua ${ }^{1,2}$ and Guo Jia-jia ${ }^{1,2}$

${ }^{1}$ Department of Pediatrics, the Third Affiliated Hospital of Zhengzhou University, Zhengzhou 450052, China ${ }^{2}$ Henan Provincial Key Laboratory of Neonatal Brain Injury, Zhengzhou 450052, China

\section{Abstract}

Objective: To investigate whether intrauterine subclinical infection sensitizes HI-induced brain injury in the immature rat, the changes and significance of Histone deacetylases (HDACs) in brain injury, and the effects of erythropoietin on White Matter injury.

Methods: Pregnant SD rats at gestation day 15 were injected LPS (0.3 mg/kg) or sterile saline (N.S) intraperitoneally, continue to rise until delivery. Rat pups on postnatal $(P)$ days 5 were randomly assigned into 4 groups: control group, LPS, HI, LPS + HI group. Intervention groups included LPS $+\mathrm{HI}+\mathrm{N}$.S group and LPS+HI+EPO group. Brain tissues were observed on the time point of $6 \mathrm{~h}, 24 \mathrm{~h}$ and $7 \mathrm{~d}$ after 40-minHI. The expression of TNF- $\alpha$ and HDACs in the brain homogenate were measured by ELISA. The level of MBP and MAP-2 were detected by immunohistochemistry staining The expression of MAP-2 mRNA and HDAC1 mRNA were detected by real time PCR.

Results: The expression of TNF-a, HDACs and HDAC1 mRNA from high to low were LPS+HI, LPS/HI, control group, but MBP was the lowest, there were significant differences between LPS+HI group and the other three groups $(P<0.05)$, there were no difference between the other three groups $(P>0.05)$. The expression of MBP in LPS+HI+EPO was higher than LPS $+\mathrm{HI}+\mathrm{N} . \mathrm{S}$, the difference was statistically significant $(P<0.05)$. There exist necrosis areas in the cortex of LPS + HI group in MAP-2 immunohistochemistry staining, but not in other three groups. Compared with the other three groups, the expression of MAP-2 mRNA in LPS $+\mathrm{HI}$ group decreased $6 \mathrm{~h}$ after HI, and gradually rise, the differences were statistically significant.

Conclusions: Intrauterine subclinical inflammation sensitizes HI-Induced injury in the immature rat brain, and lead to epigenetic changes. EPO plays a protective role to white matter after brain damage.

Keywords: Immature brain; Subclinical inflammation; Hypoxiaischemia; Epigenetic; EPO

Abbreviations: LPS: Lipopolysaccharide; HI: Hypoxic Ischemia; SS: Sterile Saline; EPO: Erythropoietin; TNF-A: Tumor Necrosis Factor A; Hdacs: Histone Deacetylases; MBP: Myelin Basic Protein; MAP-2: Microtubule-Associated Protein-2; HDAC1mRNA: Histone Deacetylases-1 Mrna; PCR: Polymerized Chain Reaction; PVL: Periventricular Leukomalacia; PWM: Periventricular White Matter; WM: White Matter; Dnmts: Methyl transferase; Hats: Histone Deacetylases; SVZ: Subventricular Zone; DG: Dentate Gyrus

\section{Introduction}

About more than one thousand premature infants were born around the world each year, average rate of premature in industrialized countries is about $9 \%-10 \%$ [1], the incidence of preterm infants was $8.1 \%$ in china. Despite the increase in the survival of very low birth weight (VLBW) preterm infants in recent years, cerebralpalsy still occurs in $10 \%$ and cognitive/behavioral deficits in $25-50 \%$ of the very preterm survivors [2]. Brain injury in premature infant mainly includes ventricle or Intraventricular Hemorrhage (PIVH) and Periventricular Leukomalacia (PVL), PVL is the major brain injury in preterm infants [3]. Epidemiology data shows, Hypoxia Ischemia (HI) and infection are the two important factors for PVL or cerebral palsy. Although single $\mathrm{HI}$ and infection is not enough to lead to brain damage, combination of both in the perinatal period may cause serious damage to immature brain tissue. There was much research about the synergy of HI combined with infection abroad, but no clear reports about whether intrauterine subclinical infection sensitizes HI-induced injury to the immature rat brain at home and abroad. Therefore, based on the animal model of intrauterine subclinical infection sensitized hypoxic-ischemic newborn mice brain injury, by detecting of the dynamic changes of Histones
Acetylation Enzyme (HDACs) and histone acetylation enzyme 1 mRNA (HDAC1mRNA), aim to investigate the possible mechanism of immature brain injury from the aspects of epigenetics.

\section{Materials and Methods}

\section{Animals}

30 Female and 10 male adult clean grade SD rats, weighing 350 g-450 g, were purchased from Experimental Animal Center of Henan Province [batch number: SCXK (prepare) 2012-2012]. Female rats were raised together with male at a ratio of 3:1 and vaginal secretion smear were detected at 80 'clock every morning. It was regarded as the $1^{\text {st }}$ day of pregnancy when vaginal plug was formed or the vision was detected full of sperm with optical microscope. The pregnant rats were kept feeding separately up to the $15^{\text {th }}$ day of gestational age.

\section{Method}

Neonatal rat model of immature brain injury: Pregnant SD rats

*Corresponding author: Xu Fa-lin, Department of Pediatrics, Third Affiliated Hospital of Zhengzhou University, Zhengzhou 450052, P.R China, Tel: 0371 6690313; E-mail: xufalin72@126.com

Received Janaury 09, 2014; Accepted February 04, 2014; Published February 06, 2014

Citation: Fa-lin X, Yan-hua Z, Jia-jia G (2014) Intrauterine Subclinical Inflammation Sensitizes Hypoxic Ischemia-Induced Injury in the Immature Rat Brain and the Mechanisms. J Neonatal Biol 3: 126. doi:10.4172/2167-0897.1000126

Copyright: (c) 2014 Fa-lin X, et al. This is an open-access article distributed unde the terms of the Creative Commons Attribution License, which permits unrestricted use, distribution, and reproduction in any medium, provided the original author and source are credited. 
at gestation day 15 were injected LPS $(0.3 \mathrm{mg} / \mathrm{kg})$ or sterile saline (N.S) intraperitoneally, continue raising until delivery. The 5-day-old pups were randomly assigned into control group (NS injection without $\mathrm{HI}$ ), LPS group (LPS injection without HI), HI group (NS injection and HI) and LPS+HI group (LPS injection and HI). The intervention group was divided into LPS+HI+NS and LPS+HI+EPO group. Unilateral HI was induced by left carotid artery ligation followed by hypoxia according to the Rice-Vannucci model [4]. Mice were anesthetized with ether, and the duration of anesthesia was $<5 \mathrm{~min}$. After surgery, the pups were allowed to recover for $1 \mathrm{~h}$. The litters were placed in a chamber perfused with a humidified gas mixture ( $8 \%$ oxygen in nitrogen) for $40 \mathrm{~min}$. The temperature in the incubator was kept at $36^{\circ} \mathrm{C}$. After hypoxic exposure, pups were returned to their biological dams. For the intervention groups, EPO $(5000 \mathrm{u} / \mathrm{kg})$ or isodose N.S was injected intraperitoneally $4 \mathrm{~h}$ before and immediately after $\mathrm{HI}$, and once a day for consecutive 5 days after $\mathrm{HI}$

The rats were sacrificed by decapitation at $6 \mathrm{~h}, 24 \mathrm{~h}$ and $7 \mathrm{~d}$ after HI. The expression of TNF- $\alpha$ and HDACs in the brain homogenate were measured by ELISA, The level of MBP and MAP-2 was detected by immunohistochemistry staining. The expression of MAP-2 mRNA and HDAC1 mRNA was detected by Real Time PCR.

ELISA: Pups were anesthetized with ether inhalation. The brains were rapidly dissected out on a bed of ice after perfused with N.S from the left ventricle. The left hemispheres were obtained with RNase free blade, weigh wet weight, homogenized after adding NS at a ratio of mass: volume $=1: 9$. Homogenate were taken to centrifuge tube and centrifuged $2000 \mathrm{r} \cdot \mathrm{min}^{-1}$ at $4^{\circ} \mathrm{C}$ for $20 \mathrm{~min}$ the supernatant were reserved for further handling. The levels of TNF and HDACs in the brain homogenate were measured according to ELISA kit instructions strictly.

Immunohistochemistry staining: After the brains had been removed and immersion-fixed in $4 \%$ paraformaldehyde at room temperatures for $24 \mathrm{~h}$, they were dehydrated with xylene and graded alcohols, paraffin embedded, serial cut into $5 \mu \mathrm{m}$ sections and mounted on salinized slides. Sections were deparaffinized and rehydrated in graded ethanol before staining. Antigen recovery was performed by heating the sections in $10 \mathrm{Mm}$ boiling citrate buffer ( $\mathrm{pH}$ 6.0) for 10 minutes. Nonspecific binding was blocked for $30 \mathrm{~min}$ with $4 \%$ goat serum in PBS. Anti-MBP, diluted 1:100 in PBS, anti-MAP-2, diluted 1:250 in PBS, was incubated at $4^{\circ} \mathrm{C}$ overnight, followed by 60 min with horse anti-goat secondary antibody. Endogenous peroxidase activity was blocked with $3 \% \mathrm{H}_{2} \mathrm{O}_{2}$ quenched by incubation with $0.3 \%$ peroxide in PBS for $5 \mathrm{~min}$. Visualization was performed using streptavidinbiotin-peroxidase complex (ABC kit).

Sections were stained for 5 minutes with DAB and counterstained with hematoxylin, dehydrated, and then mounting.

Ream Time PCR: The brains were removed and placed in $-20^{\circ} \mathrm{C}$ refrigerator for $20 \mathrm{~min}$, then cut out the left hemisphere which including the cortex, subcortical white matter and hippocampus, by RNase free blade. Total RNA was isolated using the pillar kit, agarose gel electrophoresis was carried out to detect the extraction of RNA. Experimental procedures were conducted according to the kit instructions. Reverse transcription of RNA to cDNA was carried out using Super-Script III First-Strand Synthesis Super Mix. PCR amplification consisted of 35 cycles of 30s denaturation at $94^{\circ} \mathrm{C}, 30 \mathrm{~s}$ annealing at $60^{\circ} \mathrm{C}$ and 30 s of elongation at $72^{\circ} \mathrm{C}$. For Real-Time PCR reactions, SYBR GREENPCR Master Mix was used in a PTC-200 with a Chromo 4 fluorescence detector. Primers were the follows: MAP-2: Forward-5'-GGCACTCCTCCAAGCTACTCT-3', Reverse-
5'CTTGACGTTCTTCAGGTCTGG-3', $204 \mathrm{bp}$, annealing temp 87.81 ${ }^{\circ} \mathrm{C}$; HDAC1: Forward- 5'-ACGGGGA TGTTGGAAACTACT-3', Reverse-5'-GTTGGCTTTGTGAGGACGATA-3', 134 bp, annealing temp $82.98^{\circ} \mathrm{C}, \beta$-actin: Forward-5'-CCCATCTATGAGGGTTACGC-3', Reverse-5'-TTTAATGTCACGCACGATTTC-3', 150 bp, annealing temp $82.98^{\circ} \mathrm{C}$. Results were output with $\mathrm{Ct}$ value, and calculated with relative quantitative method $\left(2^{-\Delta \Delta \mathrm{Ct}}\right)$.

\section{Statistics}

Data were expressed as mean \pm SEM. Statistical significance $(P<0.05)$ was determined by using ANOVA or t-test. $L S D$ test was used for comparing data between the two groups. All statistical analyzes were performed using SPSS 17.0 software.

\section{Results}

\section{General conditions}

There were no differences in activity, diet stillbirth rate, and premature delivery between the two pregnant rats groups. It also showed no differences in the birth weight of pups from N.S group $(6.56$ $\pm 0.33 \mathrm{~g})$ and LPS group $(6.35 \pm 0.37 \mathrm{~g})$.

\section{The TNF- $\alpha$ expression in different groups}

ELISA results showed that the TNF-immunoreactivity in the ipsilateral hemisphere of LPS+HI group was significant high than that of other three groups at $6 \mathrm{~h}$ and $24 \mathrm{~h}$ after $\mathrm{HI}(P<0.05)$. The expression of TNF- $\alpha$ have no differences between the four groups at $7 \mathrm{~d}$ after $\mathrm{HI}$ (Table 1 and Figure 1).

\section{The MBP expression in different groups at $7 \mathrm{~d}$ after $\mathrm{HI}$}

The IOD of MBP in the four groups from high to low is control group, LPS, HI and LPS+HI group at 7d after HI. Compared with the first three groups, the differences were statistically significant $(P<0.05)$, and there were no significant differences between the first three groups. The IOD of MBP in EPO intervention group was higher than that of N.S intervention group, the difference was statistically significant $(P<0.05)$ (Table 2 and Figure 2).

\section{MAP-2 immunohistochemical staining and MAP-2 mRNA expression}

The cortical neurons in control group present conical or triangular, with compact arrangement, normal cell morphology and structure, prominent nucleolus. While the neurons in LPS and HI groups at 6 $\mathrm{h}$ and $24 \mathrm{~h}$ after HI present mild edema, cellgap increased, nerve fibers manifested as funicular, but without necrosis and hemorrhage. The neurons in LPS+HI group present evident edema, the staining of cellular nuclear is pale, the nerve fibers besides the injured area partly drop out and with only the thick stained particles, peripheric small blood vessels dilated, and scattered necrotic focus with MAP-2 negative staining emerged. The morphology of cortical neuron at $7 \mathrm{~d}$ after $\mathrm{HI}$ in control group, LPS and HI group were normal, and that in LPS+HI

\begin{tabular}{|l|l|l|l|l|}
\hline group & $\mathbf{n}$ & $\mathbf{6} \mathbf{h}$ & $\mathbf{2 4} \mathbf{h}$ & $\mathbf{7 d}$ \\
\hline control & 8 & $368.64 \pm 5.70$ & $384.09 \pm 14.52$ & $389.33 \pm 16.37$ \\
\hline HI & 8 & $381.31 \pm 11.60^{\#}$ & $410.32 \pm 9.78^{\#}$ & $355.77 \pm 24.04$ \\
\hline LPS & 8 & $374.76 \pm 25.68^{\#}$ & $384.91 \pm 9.72^{\#}$ & $350.39 \pm 6.50$ \\
\hline LPS+HI & 8 & $433.20 \pm 19.74^{*}$ & $459.23 \pm 6.07^{*}$ & $354.02 \pm 12.39$ \\
\hline F & & 8.259 & 10.639 & 2.920 \\
\hline$P$ & & 0.003 & 0.001 & 0.087 \\
\hline
\end{tabular}

*contrast with control group $\mathrm{P}<0.05$, " contrast with LPS $+\mathrm{HI}$ group $\mathrm{P}<0.05$

Table 1: The level of TNF-aexpression $(x \pm s p g / m l)$. 
Citation: Fa-lin X, Yan-hua Z, Jia-jia G (2014) Intrauterine Subclinical Inflammation Sensitizes Hypoxic Ischemia-Induced Injury in the Immature Rat Brain and the Mechanisms. J Neonatal Biol 3: 126. doi:10.4172/2167-0897.1000126

Pge 3 of 6

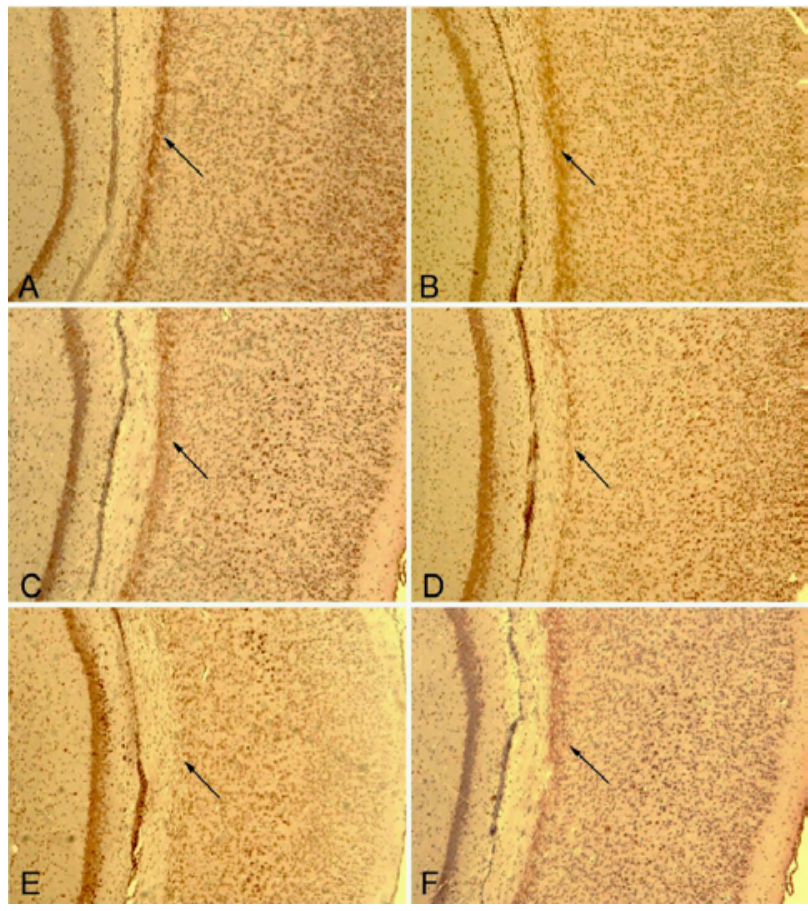

Figure 1: Immunohistochemistry stainingofMBP $(\mathrm{SP}, \times 100$, Arrow show positive cells).

\begin{tabular}{|l|l|l|l|l|l|}
\hline group & $\mathbf{n}$ & IOD & group & $\mathbf{n}$ & IOD \\
\hline Control & 8 & $140.58 \pm 4.65$ & LPS+HI+N.S & 8 & $103.36 \pm 3.62$ \\
\hline HI & 8 & $133.49 \pm 2.62$ & LPS+HI+EPO & 8 & $131.59 \pm 2.24$ \\
\hline LPS & 8 & $135.50 \pm 2.53$ & & & \\
\hline LPS+HI & 8 & $105.29 \pm 5.49^{*}$ & & & \\
\hline F & & 60.20 & $t$ & & 16.24 \\
\hline & & 0.000 & $P$ & & 0.000 \\
\hline
\end{tabular}

*contrast with other groups $\mathrm{P}<0.05$

Table 2: The IOD of MBP expression $(x \pm s)$.

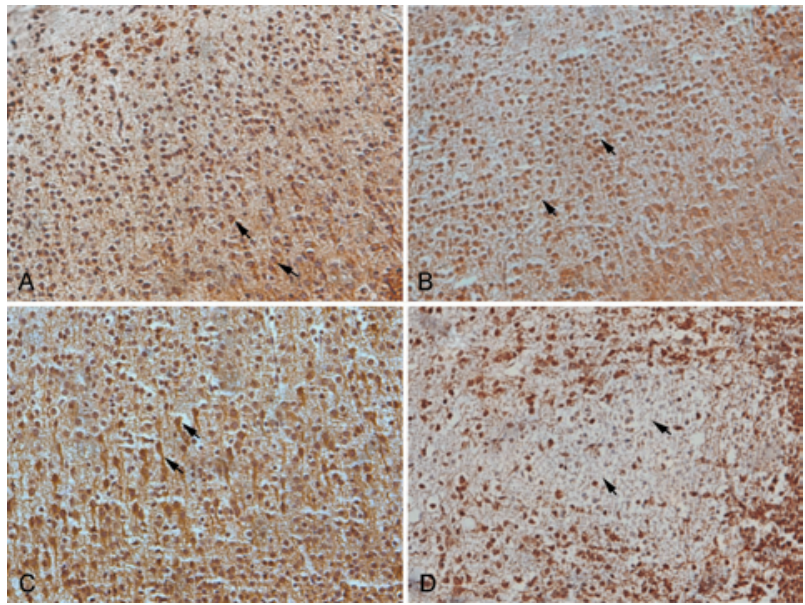

$\begin{array}{llll}\text { A. control } & \text { B. HI } & \text { C. LPS } & \text { D. LPS }+H I\end{array}$

Figure 2: Immunohistochemistry stainingofMAP-2 $24 \mathrm{~h}$ after $\mathrm{HI}(\mathrm{SP}, \times 400)$

group present as structure integrity, with no edema or necrosis, but the number of neurons was relatively reduced Figure 2.
HI group at $6 \mathrm{~h}$ after $\mathrm{HI}$ was the lowest, and has statistically significant differences $(P<0.05)$, there were no differences among the other three groups. Compared with the control and HI group, the expression of MAP- 2 mRNA in LPS+HI group was the highest at 24 $\mathrm{h}$ after $\mathrm{HI}$, the differences were statistically significant $(P<0.05)$. The level of MAP-2 mRNA at 7d after HI from high to low were: LPS+HI, LPS, HI, control group, and the differences were statistically significant $(\mathrm{P}<0.05)$ compared with the later three groups (Table 3 and Figure 3$).$

\section{HDACs comparison at different time}

The content of HDACs in rats brain tissue began to increase in LPS $+\mathrm{HI}$ group at $6 \mathrm{~h}$ after $\mathrm{HI}$, compared with the control group the difference was significant $(\mathrm{P}<0.05)$. The content of HDACs in LPS+HI

\begin{tabular}{|l|l|l|l|l|}
\hline group & $\mathbf{n}$ & $\mathbf{6} \mathbf{h}$ & $\mathbf{2 4} \mathbf{h}$ & $\mathbf{7 d}$ \\
\hline control & 8 & $1.74 \pm 0.04^{\#}$ & $1.86 \pm 0.08^{\#}$ & $2.06 \pm 0.16 \#$ \\
\hline HI & 8 & $1.68 \pm 0.03^{\#}$ & $1.87 \pm 0.11^{\#}$ & $2.29 \pm 0.11 \#^{*}$ \\
\hline LPS & 8 & $1.71 \pm 0.07^{\#}$ & $1.90 \pm 0.07$ & $2.33 \pm 0.04 \#^{*}$ \\
\hline LPS+HI & 8 & $1.38 \pm 0.10^{*}$ & $2.02 \pm 0.09^{*}$ & $6.19 \pm 0.26^{*}$ \\
\hline F & & 24.33 & 2.87 & 727.25 \\
\hline$P$ & & 0.000 & 0.081 & 0.000 \\
\hline
\end{tabular}

*contrast with control group $\mathrm{P}<0.05$, "contrast with LPS $+\mathrm{HI}$ group $\mathrm{P}<0.05$ Table 3: The expression of MAP-2 mRNA (x.s).

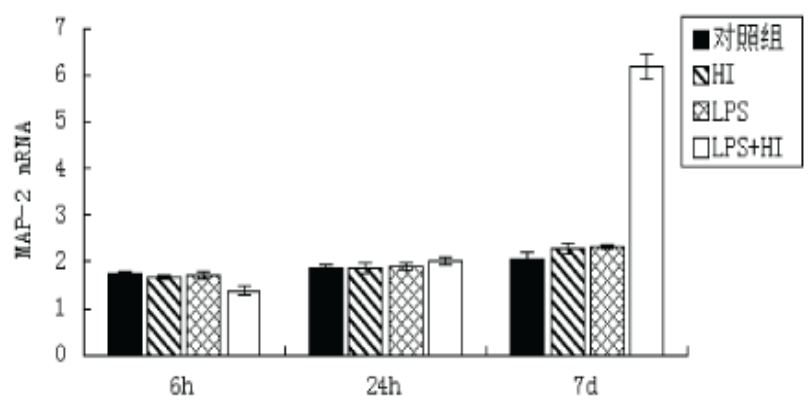

Figure 3: The value of MAP-2 mRNA.

\begin{tabular}{|l|l|l|l|l|}
\hline group & $\mathbf{n}$ & $\mathbf{6} \mathbf{h}$ & $\mathbf{2 4} \mathbf{h}$ & $\mathbf{7 d}$ \\
\hline control & 8 & $420 \pm 14.12$ & $370 \pm 16.00$ & $360 \pm 25.82$ \\
\hline HI & 8 & $505 \pm 11.35$ & $520 \pm 28.24$ & $690 \pm 18.94^{\star \#}$ \\
\hline LPS & 8 & $530 \pm 16.33$ & $465 \pm 20.21^{\#}$ & $520 \pm 16.18^{\star \#}$ \\
\hline LPS+HI & 8 & $585 \pm 20.00^{*}$ & $634 \pm 23.09^{*}$ & $1090 \pm 16.33^{*}$ \\
\hline F & & 3.206 & 7.391 & 90.886 \\
\hline$P$ & & 0.059 & 0.005 & 0.000 \\
\hline
\end{tabular}

*compare with control group $\mathrm{P}<0.05$, "compare with LPS+HI group $\mathrm{P}<0.05$

Table 4: The expression of HDACs $\left(x \pm s \mathrm{nmol} \cdot \mathrm{L}^{-1}\right)$.

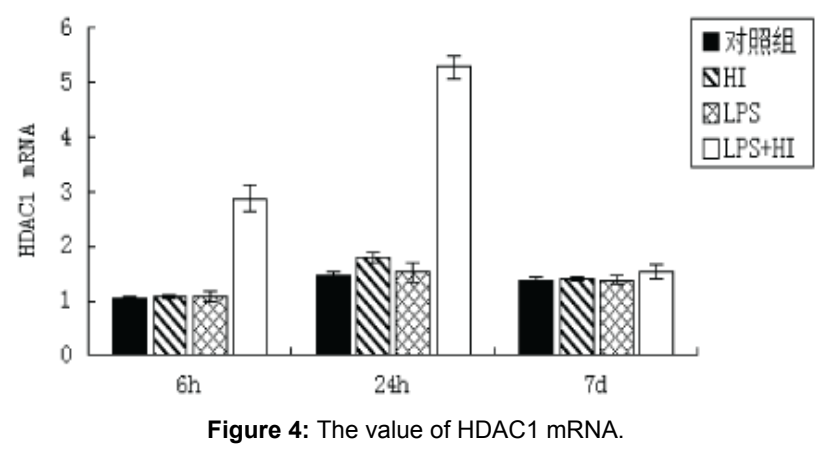


Citation: Fa-lin X, Yan-hua Z, Jia-jia G (2014) Intrauterine Subclinical Inflammation Sensitizes Hypoxic Ischemia-Induced Injury in the Immature Rat Brain and the Mechanisms. J Neonatal Biol 3: 126. doi:10.4172/2167-0897.1000126

group was the highest at $24 \mathrm{~h}$ and $7 \mathrm{~d}$ after $\mathrm{HI}$, and significant differences could be found between the LPS+HI group and the other three groups. It also showed that the content of HDACs in HI and LPS groups were higher than the control group (Table 4).

\section{HDAC1 in neonatal rat brain tissue}

HDAC1 mRNA in LPS+HI group has been significantly increased at $6 \mathrm{~h}$ after $\mathrm{HI}$, and reached the peak at $24 \mathrm{~h}$ after HI. It showed no differences among the HI group, LPS group and the control group (Table 5 and Figure 4).

\section{Real time PCR specific detection}

PCR amplification electrophoresis results reacted that the fragment consistent with the expected bands (Figure 5). The ct values was 15-35 in PCR amplification curves (Figures 6 and 8), the template concentration was appropriate. The melting curve was specific, no nonspecific products or primer dimers peak, Tm were $82.98^{\circ} \mathrm{C}, 87.81^{\circ} \mathrm{C}$ (Figures 7 and 9).

\section{Discussion}

\section{Sensitized effects of intrauterine subclinical infection on hypoxic ischemic brain damage}

Hypoxia-ischemia (HI) and inflammation in the perinatal period are the two major risk factors for brain injury in premature infants or cerebral palsy. Studies have shown that systemic LPS and HI, either given individually or in combination, can induced gray matter and white matter injury in neonatal rodents at the age of P7 [5]. Research on whether infection and hypoxia exists a synergy effect on immature brain damage, and its related mechanism has been a hotspot in perinatology. Studies have shown that low doses of LPS $(0.5 \mathrm{mg} / \mathrm{kg})$ combined with HI or LPS $(0.6 \mathrm{mg} / \mathrm{kg})$ combined with normobaric hyperoxia can cause brain damage in the neonatal rats of $\mathrm{P} 2$, mainly

\begin{tabular}{|l|l|l|l|l|}
\hline group & $\mathbf{n}$ & $\mathbf{6} \mathbf{h}$ & $\mathbf{2 4} \mathbf{h}$ & $\mathbf{7 d}$ \\
\hline control & 8 & $1.06 \pm 0.02^{\#}$ & $1.47 \pm 0.08^{\#}$ & $1.37 \pm 0.06^{\#}$ \\
\hline HI & 8 & $1.09 \pm 0.04^{\#}$ & $1.79 \pm 0.09^{* \#}$ & $1.40 \pm 0.04^{\#}$ \\
\hline LPS & 8 & $1.07 \pm 0.10^{\#}$ & $1.52 \pm 0.19^{\#}$ & $1.39 \pm 0.09^{\#}$ \\
\hline LPS+HI & 8 & $2.88 \pm 0.23^{*}$ & $5.29 \pm 0.21^{*}$ & $1.53 \pm 0.12^{*}$ \\
\hline F & & 195.50 & 585.87 & 2.98 \\
\hline$P$ & & 0.000 & 0.000 & 0.074 \\
\hline
\end{tabular}

*contrast with control group $\mathrm{P}<0.05$, " contrast with LPS $+\mathrm{HI}$ group $\mathrm{P}<0.05$ Table 5: The expression of HDAC1 mRNA $(x \pm s)$.

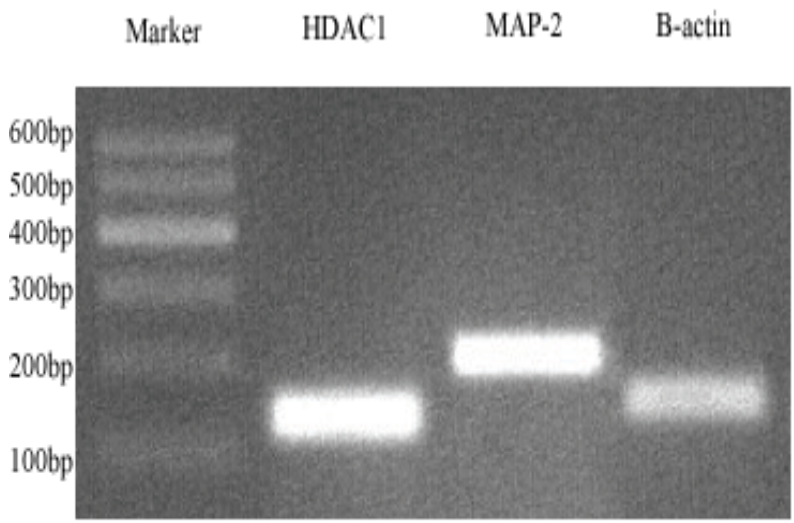

Figure 5: The RNA amplificationof HDAC1, MAP-2, $\beta$-actin.

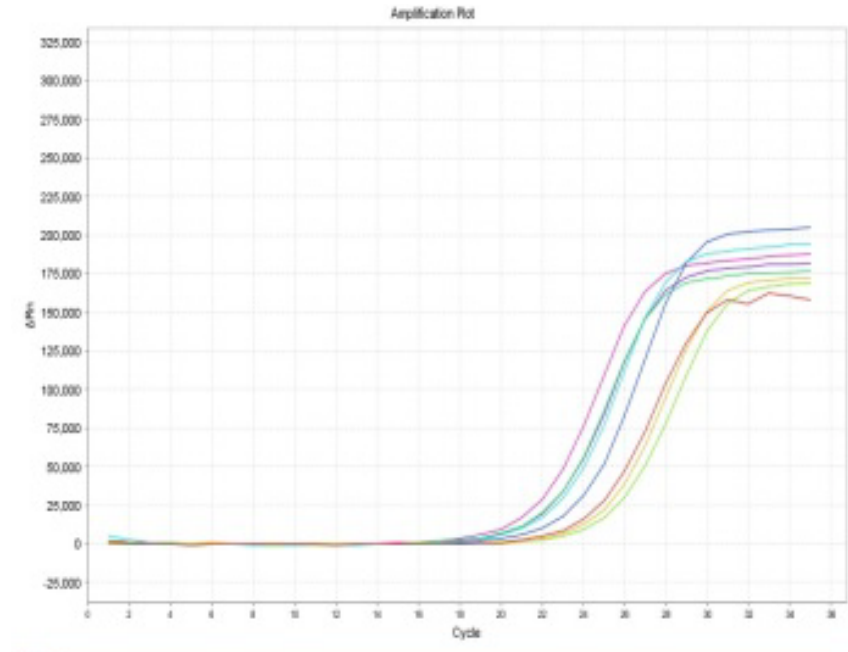

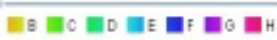

Figure 6: Amplification plot of HDAC1.

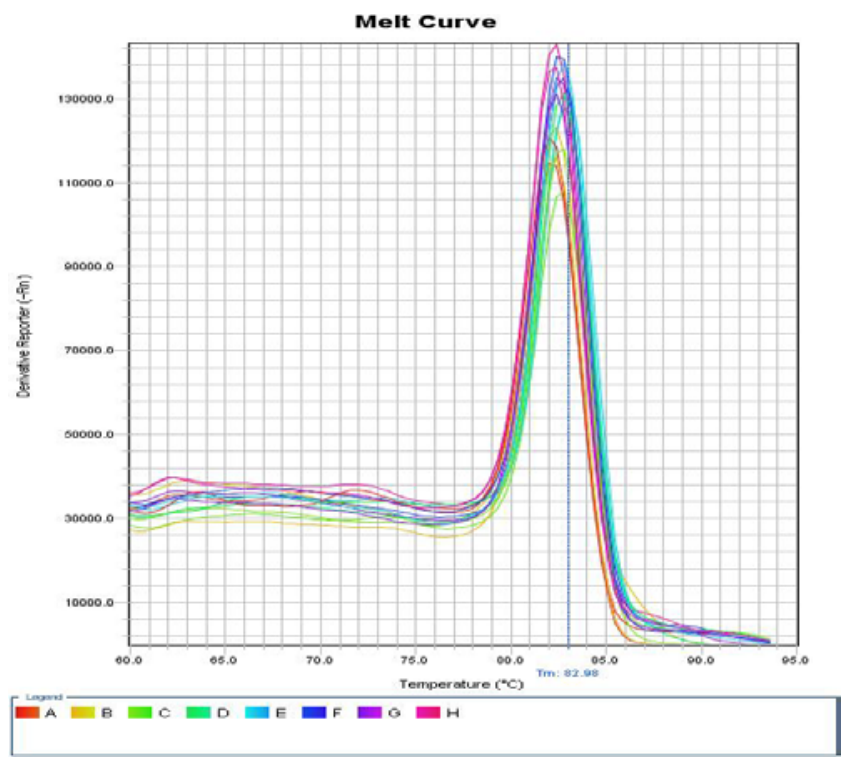

Figure 7: Melt curve of HDAC1.

by increasing white matter nerve inflammation and blood brain barrier damage, by selectively reducing the expression of MBP and the number of oligodendrocytes [6,7]. At the present study, pregnant rats were administered subclinical doses of LPS by intraperitoneal injection and pups were subjected to $\mathrm{HI}$ at postnatal day 5, and try to explore the possible interactions by observing the pathology in cortex and subcortical white matter. Our results showed that injection of LPS (0.3 $\mathrm{mg} / \mathrm{kg}$ ) intraperitoneally to the pregnant mice did not cause premature delivery or neonatal pups' birth weight decline. Intrauterine subclinical doses of LPS or a short time of postnatal HI could not cause significant damage to cortical or white matter, but the combined effect of both can cause irreversible damage to immature brain, showed increased expression of TNF- $\alpha$, MBP expression descend in subcortical white matter and cortical scattered necrosis, which initially confirmed that intrauterine subclinical inflammation sensitizes HI-Induced injury in the immature rat brain. 


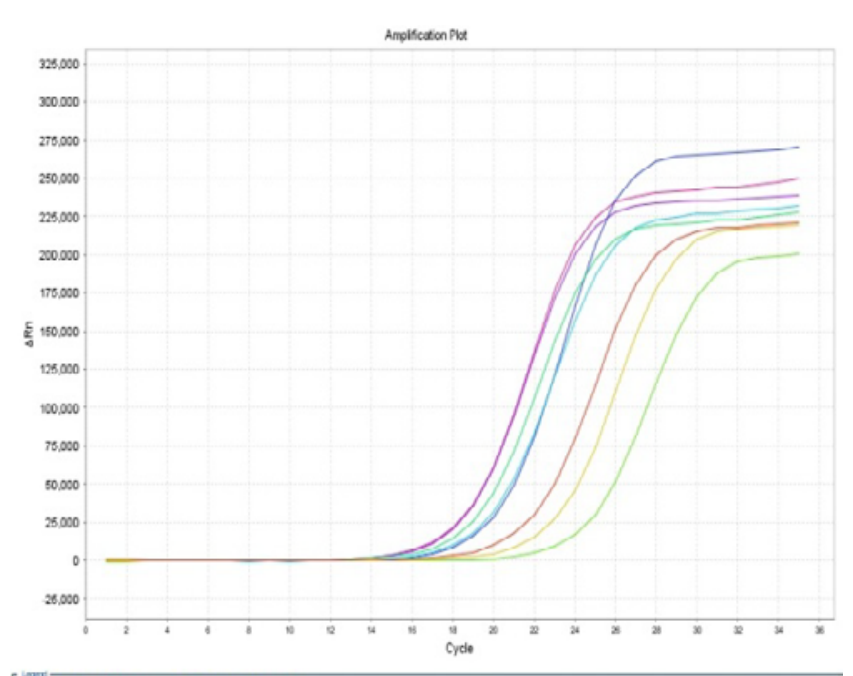

贯A

Figure 8: Amplification plot of MAP-2.

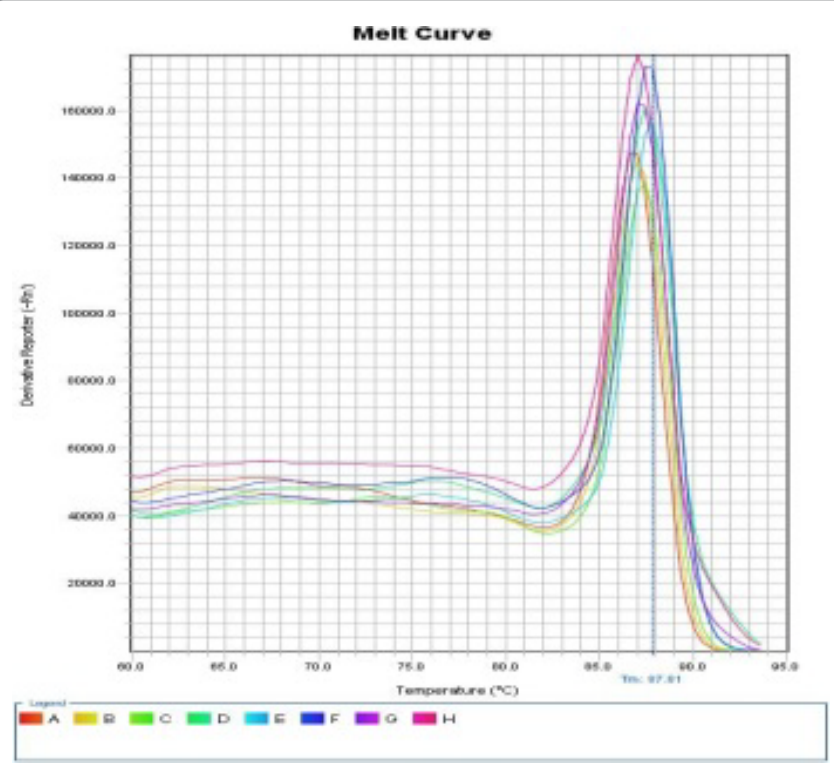

Figure 9: Melt curve of MAP-2.

TNF- $\alpha$ plays an important role in the inflammatory response, MBP is a major myelination protein. Intrauterine infection mediated through the systemic inflammatory response, causing the neuronal cell mainly oligodendrocytes which form the myelin, death or maturation disorder, and leading to brain damage [8]. Subclinical infection, also known as latent infection, refers to the pathogen invades the body, only cause the body to produce specific immune response, but cause no or only minor tissue damage, with no clinical symptoms, signs, or even biochemical changes, only can be found through immunological tests. In this study, pregnant rats were administered i.p. $0.3 \mathrm{mg} / \mathrm{kg}$ of LPS, because no premature delivery or decreased birth weight, and TNF-a levels show no significant difference between the control group and the LPS group, so the dose can be simulated intrauterine subclinical infection. At $6 \mathrm{~h}$ and $24 \mathrm{~h}$ after HI, the expression level of TNF-a in LPS+HI group was significantly higher than other groups, and difference was significant; and at 7 days after HI, MBP expression of subcortical white matter in LPS+HI group was significantly lower than the other three groups, the difference was statistically significant, confirming that LPS playing sensitizing effect on HI-induced brain injury. The mechanisms TNF- $\alpha$ induced immature brain injury have been deeply studied at home and abroad, and the increased TNF-a expression in Periventricular White Matter (PWM) oligodendrocytes was consistent with decreased MBP expression in subcortical White Matter (WM) of the immature brain [9]. The increased TNF- $\alpha$ expression at early stage was significantly correlated with decreased MBP expression at later stage subcortical $\mathrm{WM}$ in this study, indicated that TNF- $\alpha$ plays an important role in perinatal PWM injury.

MAP-2 is one of the structural proteins of microtubules, and plays a key role in the neurons repair process, diffuses distribution neurons and dendrites in the cortex and CA1, CA3 and dentate gyrus of hippocampal, showing continuous fiber strips cords [10]. MAP-2 is more sensitive to injury, reacts at early stages and last for a longer time. The MAP-2 immunohistochemistry showed that scattered cortical necrosis can be found at $24 \mathrm{~h}$ after Hi in the LPS+HI group and but no obvious damage in LPS or HI group, which further confirmed intrauterine infection has an sensitizing effect on HI-induced brain injury. At gene level, MAP-2 mRNA expression significantly decreased at $6 \mathrm{~h}$ after injury, gradually returned to control levels at $24 \mathrm{~h}$, and expression quantity was significantly increased at $7 \mathrm{~d}$ after $\mathrm{HI}$, which was basically consistent with MAP-2 immunohistochemical staining. Timeliness changes of MAP-2 mRNA may be associated with inadequate blood supply locally, inhibiting microtubule assembly in early brain injury, and nerve cells in surrounding area transcribed actively, which involved in repair processes in the late period of injury.

PVL is the main pathological feature in preterm infants' brain injury, also the risk factor for cerebral palsy, yet short of specific therapeutic measures at present. It has been reported that, EPO and its derivatives CEPO have a neuroprotective effect in a variety of brain injury models. EPO was administered to the model of infection sensitized HI-induced immature brain injury in our study to investigate whether EPO has a protective effects on subcortical WM, the results showed that the expression quantity of MBP in EPO group was significantly higher than that of the control group, which initially proved that EPO has a protective effect on WM in immature animals, but its exact mechanism still needs further study.

\section{Epigenetic modification and the immature brain injury}

Premature brain injury and the following sequelae are the results of interacting of genetic and environmental factors, any diseaserelated gene expression variations can be caused by changes in the DNA sequence, also caused by the gene expression changes without the DNA sequence changes, the epigenetic modification. Epigenetic modifications include DNA modification (such as methylation), histone modifications (such as histone methylation, acetylation, etc.) and non-coding RNA, mainly related to the following key enzyme: DNA methyltransferase (DNMTs), HDACs and Histone Deacetylases (HATs). Histone acetylation is regulated by HATs and HDACs, HDACs remove acetyl group of the histone amino terminus, to increase the interaction between DNA and histone and repress transcription, and HATs the promote transcription. Embryos epigenetic programming process is vulnerable to infection, hypoxia and environmental factors, and the error of the methylation patterns can cause a variety of serious diseases [11]. More and more studies show that HDACs played a key role in the regulation of nerve function, and HDAC1 has a specific high expression in the neuronal cell bodies and axons of cortex, corpus 
Citation: Fa-lin X, Yan-hua Z, Jia-jia G (2014) Intrauterine Subclinical Inflammation Sensitizes Hypoxic Ischemia-Induced Injury in the Immature Rat Brain and the Mechanisms. J Neonatal Biol 3: 126. doi:10.4172/2167-0897.1000126

callosum, hippocampus and subcortical white matter of immature brain [12], and has a major impact for learning and memory in rats [13], therefore, HDACs and HDAC1 mRNA were chose for further study in this experiment. The experimental results showed, HDACs expression was upregulation after the immature brain injury, and maintaining higher continued until postnatal 12 days. At gene level, HDAC1 mRNA expression has significantly increased at early stage of injury and prior to the high expression of HDACs, indicating that the HDAC1 stress was very quickly to $\mathrm{HI}$ injury, which is consistent with the report of Niu Xinetc [14]. Therefore, intrauterine infection sensitized HI-induced immature brain injury may be associated with high HDACs and HDAC1 mRNA expression. Studies have showed that HDACs inhibitors can promote the proliferation and differentiation of neural stem cell, can reduce inflammation reaction and infarct size post-HI insults, improve neurobiological behavior after middle cerebral artery occlusion $[15,16]$. Thus, it can provide ideas and methods to prevent and treat preterm brain injury through the further research on epigenetics. Although HDAC1 has a major impact on Sub Ventricular Zone (SVZ) and Dentate Gyrus (DG) [17], the research on the mechanism of how histone acetylation and deacetylation regulate gene transcription is still at the primary stage, and it is unknown about the specific nervous cells that HDACs express and its expression pattern. The experiment preliminary explores the dynamic variation of HDACs and HDAC1 mRNA expression in cortex in immature animal brain injury post-HI, and provide a basis for further exploring the regulatory function of HDACs family genes on immature brain injury, also offer guidance for prevention of preterm infant brain injury in the future.

\section{References}

1. Beck S, Wojdyla D, Say L, Betran AP, Merialdi M, et al. (2010) The worldwide incidence of preterm birth: a systematic review of maternal mortality and morbidity. Bull World Health Organ 88: 31-38.

2. Vincer MJ, Allen AC, Joseph KS, Stinson DA, Scott H, et al. (2006) Increasing prevalence of cerebral palsy among very preterm infants: a population-based study. Pediatrics 118: e1621-1626.

3. Burd I, Balakrishnan B, Kannan S (2012) Models of fetal brain injury, intrauterine inflammation, and preterm birth. Am J Reprod Immunol 67: 287-294.
4. Rice JE, Vannucci RC, Brierley JB (1981) The influence of immaturity on hypoxic? ischemic brain damage in the rat. Ann Neurol 9: 131-141.

5. Svedin P, Hagberg H, Sävman K, Zhu C, Mallard C (2007) Matrix metalloproteinase-9 gene knock-out protects the immature brain after cerebral hypoxia-ischemia. J Neurosci 27: 1511-1518.

6. Brehmer F, Bendix I, Prager S, van de Looij Y, Reinboth BS, et al. (2012) Interaction of inflammation and hyperoxia in a rat model of neonatal white matter damage. PLoS One 7: e49023.

7. FalinXu,JiajiaDuan,Ju Wang (2013) Effects of bacterial lipopolysaccharide and normobarichyperoxia on immature brain development of neonatal rat. Chin J Clin Pediatr 28:110-114.

8. Yang LJ, Wang J, Tian ZF, Yuan YF (2013) Shenfu injection attenuates neonatal hypoxic-ischemic brain damage in rat. Neurol Sci 34: 1571-1574.

9. Berger I, Peleg O, Ofek-Shlomai N (2012) Inflammation and early brain injury in term and preterm infants. Isr Med Assoc J 14: 318-323.

10. Xu Wu, Li Yang, Baojie Wang (2011) An experimental study on expression of MAP-2 protein and mRNA after traumatic brain injury in rats. Chinese Journal of Forensic Medicine 26: 269-272.

11. Galle AA, Jones NM (2012) The neuroprotective actions of hypoxic preconditioning and postconditioning in a neonatal rat model of hypoxicischemic brain injury. Brain Res.

12. Morris MJ, Monteggia LM (2013) Unique functional roles for class I and class II histone deacetylases in central nervous system development and function. Int J Dev Neurosci 31: 370-381.

13. Bahari-Javan S, Maddalena A, Kerimoglu C, Wittnam J, Held T, et al. (2012) HDAC1 regulates fear extinction in mice. J Neurosci 32: 5062-5073.

14. XinNiu,YangWang,Junhui Yin (2012) The expression changes of HDAC genes in rat brain stroke. Experimental and Laboratory Medicine. 30: 4-6.

15. Baltan S, Murphy SP, Danilov CA, Bachleda A, Morrison RS (2011) Histone deacetylase inhibitors preserve white matter structure and function during ischemia by conserving ATP and reducing excitotoxicity. J Neurosci 31: 39903999.

16. Chen S, Sang N (2011) Histone deacetylase inhibitors: the epigenetic therapeutics that repress hypoxia-inducible factors. J Biomed Biotechnol 2011: 197946

17. Foti SB, Chou A, Moll AD, Roskams AJ (2013) HDAC inhibitors dysregulate neural stem cell activity in the postnatal mouse brain. Int J Dev Neurosci 31 434-447. 